\title{
Pragmatic Frequency Domain Equalization for Single Carrier with Offset Modulations
}

\author{
Miguel Luzio, Rui Dinis, Member, IEEE, and Paulo Montezuma
}

\begin{abstract}
Offset signals can be designed to have reduced envelope fluctuations allowing high amplification efficiency. When combined with SC-FDE (Single-Carrier with Frequency-Domain Equalization) schemes, they become excellent candidates for broadband wireless systems with severe power constraints. However, the performance of these modulations with conventional FDE receivers is sub-par, even for receivers specifically designed for offset modulations. This is especially serious when large offset QAM (Quadrature Amplitude Modulation) constellations are used. In this paper we consider SC-FDE schemes combined with offset modulations and study the reason behind the poor performance of conventional FDE implementations. We also present pragmatic FDE receivers for offset modulations that have low complexity and excellent performance.
\end{abstract}

Index Terms-Offset modulations, single carrier, FDE, IQI, iterative receivers.

\section{INTRODUCTION}

B ROADBAND wireless systems are expected to have high power and spectral efficiency, and to sustain high bit rates, even when subjected to severely time-dispersive channels. Future systems must be able to surpass these challenges using advanced equalization techniques that compensate the high level of signal distortion. The high channel impulse response lengths, inherent to severely time-dispersive channels, make time-domain equalization techniques inadequate, since their complexity grow linearly with the length of the channel impulse response [1]. This justifies the use of block transmission techniques combined with Frequency Domain Equalization (FDE) [2] where the complexity per data symbol is lower and less dependent from the length of the channel impulse response. At the uplink transmission, the challenge is further increased by the limited power consumption of the Mobile Terminals (MT). To preserve battery power, the MT should resort to low cost, highly efficient power amplification

Manuscript received October 18, 2012; revised February 15, 2013; accepted April 30, 2013. The associate editor coordinating the review of this paper and approving it for publication was $\mathrm{Z}$. Wang.

This work was supported by FCT / MEC (CTS multi-annual funding project PEst-OE / EEI / UI0066 / 2011, IT multi-annual funding project PEst-OE / EEI / LA0008 / 2013, MPSat project PTDC / EEA-TEL / 099074 / 2008 ADCOD project PTDC / EEA-TEL / 099973 / 2008 and doctoral grant SFRH / BD / 66172 / 2009

M. Luzio is with the Dep. de Eng. Electrotécnica, Faculdade de Ciências e Tecnologia, Universidade Nova de Lisboa, Caparica, Portugal, IT, Instituto de Telecomunicações, Portugal, and Uninova - CTS, Universidade Nova de Lisboa, Portugal (e-mail: jmluzio@gmail.com).

R. Dinis is with the Dep. de Eng. Electrotécnica, Faculdade de Ciências e Tecnologia, Universidade Nova de Lisboa, Caparica, Portugal and IT, Instituto de Telecomunicações, Portugal.

P. Montezuma is with the Dep. de Eng. Electrotécnica, Faculdade de Ciências e Tecnologia, Universidade Nova de Lisboa, Caparica, Portugal, and Uninova - CTS, Universidade Nova de Lisboa, Portugal.

Digital Object Identifier 10.1109/TWC.2013.072513.121624 and concentrate the signal processing load at their Base Stations (BS) [2], [3].

To achieve a low cost and highly efficient power amplification we should employ grossly nonlinear power amplifiers. These amplifiers are only recommendable for signals with constant or quasi-constant envelope. Offset modulations are particularly interesting in this context because both the envelope fluctuations and dynamic range of the transmitted signals are typically much lower than those of their corresponding non-offset signals. However, this is achieved at the expense of bandwidths wider than the minimum Nyquist band. Modulations like Minimum Shift Keying (MSK) [4], Gaussian MSK and other Constant Phase Modulation (CPM) schemes [5] can be decomposed as the sum of OQPSK components [6], and we can design OQPSK-type signals with good trade-offs between power efficiency, spectral efficiency and reduced envelope fluctuations [7], [8]. Offset modulations based on larger constellations such as OQAM (Offset Quadrature Amplitude Modulation) [9], while having much higher envelope fluctuations, can also be written as a sum of OQPSK components with very low envelope fluctuations. These components can then be separately amplified, without added distortion, by several grossly nonlinear amplifiers [10], allowing efficient power amplification.

SC-FDE (Single-Carrier with Frequency-Domain Equalization) schemes [11] are excellent candidates for the uplink of broadband wireless systems where MTs have strict power constraints. In fact, the achievable performance and and overall signal processing complexity are similar to OFDM systems, but the transmitted signals have much lower envelope fluctuations and the signal processing load is shifted to the receiver (the BS in the uplink case). The performance can be further improved when SC-FDE schemes are combined with efficient nonlinear equalization techniques [12]. However, when conventional FDE receivers (designed for non-offset modulations) are employed with offset modulations, the performance is very poor due to the residual interference between the in-phase and quadrature components at the sampling instants [13]. For this reason, FDE receivers specially designed for offset modulations were proposed in [13]. The basic idea behind these schemes is to design the FDE in such a way that the overall impulse response at its output (including the channel and transmit and receive filters) becomes real, avoiding IQI (In-phase/Quadrature Interference). Since these schemes can have very high residual ISI, modified FDE receivers were proposed in [14] that minimize the overall residual ISI plus IQI levels, allowing an improved performance. Unfortunately, even the best FDE receivers for offset modulations have a somewhat 
disappointing performance when large offset constellations are employed [15].

Contrary to what could be expected, the performance of offset modulations with conventional FDE receivers improves when we employ raised-cosine pulses with close to zero rolloff (i.e. with the minimum Nyquist bandwidth) [16]. In this paper, we take advantage of this particular detail to define pragmatic FDE receivers for offset modulations that can take full advantage of the multipath diversity. To decrease the complexity of the feedback loop, the oversampling and offset procedures are treated separately from the equalization process in a special block pair. The proposed FDE schemes can equalize oversampled and non-oversampled, offset and nonoffset signals alike, allowing good performance, even for high order constellations

This paper is organized as follows: section II explains both QAM (Quadrature Amplitude Modulation) and OQAM signals and their respective oversampling. Section III describes several linear receiver designs for offset modulations. A set of performance results is shown in section IV. Section V shows the designs' adaptation to iterative receivers and devises a less complex iterative method. A series of simulations were conducted to evaluate the performance of the proposed iterative receivers. The corresponding Bit-Error-Rate (BER) performance results are then presented in section VI. A complexity analysis is made in section VII and section VIII gives the final remarks for this paper.

\section{OFFSET QAM SIGNALS}

Let us consider an SC-FDE scheme where the data is transmitted in blocks of $N$ symbols with a suitable cyclic prefix of $N_{C P}$ samples appended to the beginning of each block. The data block to be transmitted is $\left\{a_{n} ; n=0,1, \ldots, N-1\right\}$, where $a_{n}=a_{n}^{I}+j a_{n^{\prime}}^{Q}$ is the $n$th data symbol, $a_{n}^{I}$ for the in-phase component and $a_{n}^{Q}$ for the quadrature component. The data bits of an $M^{2}$-OQAM constellation with no zero crossings and low envelope fluctuations with a general grey mapping can be described as,

$$
a_{n}^{I}=\sum_{p=1}^{P} \varphi^{(p)} \prod_{m=1}^{p} b_{n}^{I(m)},
$$

for the in-phase component and

$$
a_{n}^{Q}=\sum_{p=1}^{P} \varphi^{(p)} \prod_{m=1}^{p} b_{n}^{Q(m)}
$$

for the quadrature component, where $b_{n}^{I(m)}= \pm 1$ and $b_{n}^{Q(m)}= \pm 1$ are the $m$ 's in-phase and quadrature sent data bits [17], [18],

$$
P=\log _{2}(M),
$$

and $\left\{\varphi^{(p)} ; p=1, \ldots, P\right\}$ is the resolution block $\left(\varphi^{(1)}\right.$ is the highest order resolution bit and $\varphi^{(P)}$ the lowest). For uniform constellations, $\varphi^{(p)}=2^{P-p}$. This implies that the values associated to $a_{n}^{I}$ and $a_{n}^{Q}$ for an 4-OQAM constellation are \pm 1 , for 16 -OQAM \pm 1 and \pm 3 , for 64 -OQAM $\pm 1, \pm 3, \pm 5$, and \pm 7 and so forth.
Assuming that the complex envelope of the transmitted signal is

$$
x(t)=\sum_{n=-N_{C P}}^{N-1} a_{n}^{I} r\left(t-n T_{s}\right)+j a_{n}^{Q} r\left(t-n T_{s}-T_{o}\right),
$$

where $r(t)$ is the adopted pulse shape, $T_{s}$ is the sampling time, $T_{o}=T_{s} \phi$ is the time offset between both I and Q components, usually with $\phi=0.5$, and $N_{C P}$ is the length of the cyclic prefix required for an efficient FDE implementation [2]. The block $\left\{a_{n} ; n=0,1, \ldots, N-1\right\}$ is periodic with period $N$. Therefore, the cyclic prefix is a repetition of the last $N_{C P}$ data symbols of the data block, $a_{-n}=a_{N-n}$.

At the receiver side, if the received block is sampled at $T_{s} / J$ rate, with $J \in \mathbb{N}$ samples per symbol, the samples associated to the useful part of the block, without cyclic prefix, will be $\left\{x_{n}^{(J)} ; n=0,1, \ldots, J N-1\right\}$, with $x_{n}^{(J)} \triangleq x\left(n T_{s} / J\right)^{1}$. Since $x(t)$ is cyclostationary [19], $E[x(t) x(t-\tau)]$ is periodic in $t$, with period $T_{s}$ for non-offset modulations, and $T_{s} / 2$ for offset modulations.

The frequency-domain block associated to $\left\{x_{n}^{(J)} ; n=\right.$ $0,1, \ldots, J N-1\}$, can be defined as $\left\{X_{k}^{(J)} ; k=\right.$ $0,1, \ldots, J N-1\}$, and

$$
X_{k}^{(J)}=A_{k}^{(J)} R_{k}^{(J)}
$$

where

$$
\begin{aligned}
& \left\{R_{k}^{(J)} ; k=0,1, \ldots, J N-1\right\}= \\
& \quad \mathbf{D F T}\left\{r_{n}^{(J)} \triangleq r\left(n T_{s} / J\right) ; n=0,1, \ldots, J N-1\right\},
\end{aligned}
$$

and

$$
\begin{aligned}
&\left\{A_{k}^{(J)} ; k=0,1, \ldots, J N-1\right\}= \\
& \operatorname{DFT}\left\{a_{n}^{(J)} ; n=0,1, \ldots, J N-1\right\},
\end{aligned}
$$

as the oversampled data symbols. The relation of the oversampled data symbols to the regular data symbol block is distinct whether we consider offset or non-offset modulations.

\section{A. Non-offset Modulations}

If we consider a non-offset modulation $(\phi=0)$, the relation of the oversampled data symbols to the regular data symbol block, as we can see in Fig. 1, is

$$
a_{n}^{(J)}=\left\{\begin{array}{cc}
a_{n^{\prime}}, & n=J n^{\prime} \\
0, & \text { otherwise }
\end{array},\right.
$$

with $n=0,1, \ldots, J N-1$ and $n^{\prime}=0,1, \ldots, N-1$. In the frequency domain, the correspondent oversampled data symbol block is

$$
A_{k}^{(J)}=A_{k \bmod N},
$$

with $x \bmod y$ as the remainder of division of $x$ by $y$. The process to obtain the regular data symbols from an non-offset oversampled frequency data block is

$$
\begin{aligned}
& \left\{a_{n} ; n=0,1, \ldots, N-1\right\}= \\
& \quad \text { IDFT }\left\{\sum_{q=0}^{J-1} A_{k+q N}^{(J)} ; k=0,1, \ldots, N-1\right\}
\end{aligned}
$$

\footnotetext{
${ }^{1} J$ is assumed to be large enough to avoid aliasing effects.
} 